\title{
Management of Familial Hypercholesterolaemia in Childhood
}

\author{
Uma Ramaswami ${ }^{1}$ \& Steve E Humphries ${ }^{2}$ \\ ${ }^{1}$ Lysosomal Disorders Unit, Royal Free Hospital, London. \\ ${ }^{2}$ Centre for Cardiovascular Genetics, University College London, London;
}

Corresponding author: Professor Steve E Humphries, Institute of Cardiovascular Science, The Rayne Building, University College London, London, UK WC1E 6JF, Tel 0207679 6962 email: steve.humphries@ucl.ac.uk

\section{Abstract}

Purpose of review: All guidelines for the management of heterozygous Familial hypercholesterolaemia (FH) in children and young people recommend statins to lower Low Density Lipoprotein Cholesterol (LDL-C) concentrations, to reduce the individual's adult risk of developing cardiovascular disease (CVD). Here we review recent findings regarding the efficacy and safety of the use of stains in childhood.

Recent findings: As expected from their safety profile in adults, there is no evidence from short term trials or long term follow up that statin use in children is associated with any adverse effects on growth, pubertal development or muscle or liver toxicity. Long-term follow-up indicates benefits with respect to lower CVD rates. Factors that influence adherence are discussed, as is the role of the underlying genetic causes for $\mathrm{FH}$ and of variation at other genes in determining the LDL-C lowering effect.

Summary: Based on the good safety profile, and the expert opinion guidelines, clinicians should consider prescribing statins for children with $\mathrm{FH}$ from the age of at least 10 years (and earlier if CVD risk is particularly high in the family). Up- titrating statin dosage and the use of additional lipid-lowering therapies should be considered so that LDL-C concentrations are lowered to recommended targets. 198 words

Keywords: Treatment Guidelines, Statin safety, SCLO1B1 genotype, adherence, 


\section{Introduction}

Familial Hypercholesterolaemia (OMIM \#143890) is an autosomal dominant disease which results in elevated concentrations of atherogenic Low Density Lipoprotein-cholesterol (LDL$\mathrm{C}$ ) in the blood from birth (or possible even in utero). Individuals with $\mathrm{FH}$ have a much higher risk of developing premature cardiovascular disease (CVD) than the general population. In a follow-up study of over 14,000 subjects with clinical characteristic of $\mathrm{FH}$, matched with 42,500 UK General practice individuals (mean age 45 years), those with the characteristics of FH had a Hazard Ratio (HR) 13.5 fold higher for developing CVD over a 13.8 median years follow up, than the non FH group (1). By contrast, in those with a formal diagnosis of $\mathrm{FH}$ in their notes and who were being treated with statin therapy, the CVD HR was 1.66. Data from the UK "Simon Broome" FH register (2) has shown that compared to the general population of England and Wales, male patients with "Severe" FH in the age range 20-39 years had a Standardised Mortality Ratio (SMR) of over 1700, with the SMR in women of this age being twice as high (Figure 1). For both men and women the SMR decreases with age but is always higher in women. Analysis of recent mortality data (2008-2016) has shown that with the availability of potent statins the overall (combing all ages) SMR [95\% Cl] has fallen in men from the pre-statin era (before 1992) of 356 [178 -637] to 159 [91-258] while in women the fall has been much less 498 [215-982] to 350 [192-588]. Overall these two papers confirm the significantly higher CVD and CHD risk in untreated individuals with $\mathrm{FH}$ and in particular that risk in the younger age group and especially in women remains high even with the availability of potent statins.

Long-term placebo-controlled RCTs are neither ethical nor feasible in those with FH because of their high CVD risk. , However, there is overwhelming evidence that children with $\mathrm{FH}$ have a markedly elevated risk of future morbidity and mortality from coronary heart disease because of their elevated LDL-C, and that early identification and treatment to lower their LDL-C will concomitantly reduce this risk. Based on the population prevalence of $\mathrm{FH}$ as $\sim 1 / 250$ (3), there are approximately 50,000 FH children under 18 years in the UK, 260,000 in the USA and over 400,000 in the EU. Over recent years there have been many consensus and expert opinion-based guidelines for the management of children with heterozygous $\mathrm{FH}$ with the European Atherosclerosis society guideline published in 2014 (4) and a UK guideline in 2019 (5). All guidelines recommend that children identified with FH should receive clear healthy lifestyle advice, which should of course be also adopted by the whole family. This includes healthy diet choices as well as exercise and non-smoking information. All guidelines also recommend the use of statin lipid-lowering therapy as a first line treatment, and this review focusses on the recommendations, safety issues and lifestyle and genetic determinants of response to statin therapy that have been published in the last 18 months for the treatment of children with heterozygous FH.

\section{Treatment and safety}

The UK consensus statement on the management of children and young people with heterozygous $\mathrm{FH}$ proposes management strategies for those identified by cascade testing 
when a parent is diagnosed with $\mathrm{FH}$ and for those diagnosed following incidental lipid tests, including those found as infants by universal screening (5). All children should be seen in a child-friendly and child appropriate environment, by a Health Care professional (HCP) with expertise in managing children. Life-style dietary and exercise advice should be started in childhood, and should ideally be adopted by the whole family. The treatment strategy in three key age categories is shown in Figure 2. In particular, in those under 10 years of age, if drug treatment is considered appropriate, aim for a $30-50 \%$ reduction in LDL-C from baseline or a level $<3.5 \mathrm{mmol} / \mathrm{L}$, while in those over 10 years aim for a $50 \%$ reduction in LDL$C$ from baseline or a level $<3.5 \mathrm{mmol} / \mathrm{L}$. Finally in those over 14 years, if there are additional co-morbidities (e.g, type 1 diabetes) or a very strong family history of early coronary events in adults in their 2nd and 3rd decade, assuming compliance to statin therapy, the guideline suggests slowly increasing the statin dose and/or with the addition of ezetimibe $10 \mathrm{mg}$ daily, aiming towards a target LDL-C concentration of $<2.5 \mathrm{mmol} / \mathrm{I}$ over the next $3-5$ years.

The guideline contains a clear statement that boys and girls both need to be considered for lipid-lowering therapy at the same age, and that initiation of treatment should not be delayed in girls. The increased risk of CVD in FH is the result of having an increased "LDL-C burden" since even a modest elevation in LDL-C concentration accumulates over time and accelerates atherosclerosis. A recent study from Norway (6) indicates that this LDL-C burden may be greater in girls than in boys. Children and young people with $\mathrm{FH}$ ( 438 girls, 452 boys) not receiving lipid-lowering therapy, aged 0 to 19 years were included. Compared to boys, girls had significantly higher mean [95\% confidence interval] LDL-C concentrations with differences of 0.39 [0.19-0.59] $\mathrm{mmol} / \mathrm{L}(p<0.001)$. This estimate did not change after adjustment for age. This would contribute an additional $\sim 7 \%$ to the total lifelong LDL-C burden in $\mathrm{FH}$ women, and with the addition of added burden due to statin cessation during pregnancy and breastfeeding, would help explain the high CVD risk in adult women with FH.

The largest study of children published to date is the IAS Register of $>3000$ children with FH recruited from eight countries in Europe (7). Some countries such as Holland and Greece have mature FH pediatric programmes, while others (eg Austria) have only recently started systematic case-finding programmes. Not surprisingly the age of $\mathrm{FH}$ diagnosis in children varied significantly across the countries, as did the proportion of those $>10$ years being treated with statin and/or ezetimibe. Approximately a quarter of the treated children and almost three quarters of the untreated children older than 10 years still had LDL-C concentrations over the guideline recommended $3.5 \mathrm{mmol} / \mathrm{l}$. These data suggest that many children with $\mathrm{FH}$ are not receiving the full potential benefit of early identification and appropriate lipid-lowering treatment according to recommendations. Data on use of ezetimibe as an adjunct to statin therapy was particularly relevant. Ezetimibe is recommended for adults with $\mathrm{FH}$ who are statin intolerant or who fail to reach target on statin alone and for children over the age of 10 years. Ezetimibe was used as an adjunct to statin therapy in all countries but at a low and varying frequency. This might have been caused by the relatively high price at the time of analysis and because of relatively limited evidence of its use in children. However, as expected, ezetimibe use lowered LDL-C 
significantly and more than $90 \%$ of children taking a statin plus ezetimibe achieved LDL-C below $3.5 \mathrm{mmol} / \mathrm{l}$, compared to only $53 \%$ of those on statin only.

One potential harm of the identification and treatment of children with $\mathrm{FH}$ would be if statin use at an early age (eg. age 10 years) were associated with any long term safety issues. Statins have been used in adults (with $\mathrm{FH}$ and in those in the general population) since the late 1980s, and in the ensuing 40 years no major long term safety issue have been identified (8). Studies in children have also found no long term safety concerns. The 2019 Cochrane review update on the safety of statins in children (9) included 26 potentially eligible studies, which included nine randomized placebo-controlled studies (1177 participants). The magnitude of LDL-C lowering varied from study to study, most likely due to different statins and doses and possibly due to different definitions about true monogenic heterozygous FH. The review did not identify any clinically significant side effects with statins (ie abnormal liver transaminase or creatine kinase values). Sexual maturation was similar to normal population groups. The review concluded that while long term safety remains unknown, statin use in childhood was safe in the medium term. This finding is fully supported by the UK FH Children's Register (10) and by a study from France (11), both of which found no instances of clinically relevant safety issues and an equal growth rate in statin treated and non-treated children.

The issue of long term safety has been recently examined in a 20-year follow-up study of statin therapy in children (12). The individuals studied were FH child participants in a 19971999 placebo-controlled trial evaluating the 2-year efficacy and safety of pravastatin, who were now invited for follow-up, together with their 95 unaffected siblings. The new study showed that initiation of statin therapy during childhood slowed the progression of carotid intima-media thickness (CIMT) and reduced the risk of CVD in adulthood. Mean progression of CIMT over the entire follow-up period was $0.0056 \mathrm{~mm}$ per year in those with $\mathrm{FH}$ and $0.0057 \mathrm{~mm}$ per year in siblings, showing that treatment had reduced the progression of atherosclerosis in the carotid artery (and presumably also in the coronary arteries) to that of a non-FH individual. The cumulative incidence of CVD events and of death from CVD at 39 years of age was lower among the treated cohort than among their affected parents ( $1 \%$ vs. $26 \%$ and $0 \%$ vs. $7 \%$, respectively). Crucially, there were no serious adverse events reported, including no cases of rhabdomyolysis, with no significant differences in liver function observed between those with $\mathrm{FH}$ and their unaffected siblings (12).

\section{Adherence/ support}

One of the key issues in the decision as to whether, and when, to start or up-titrate statin treatment for a child, are the views of the parents/guardians about the risks associated with untreated $\mathrm{FH}$ and the potential benefit and concern about side effects of the medication prescribed. These issues have been reviewed in a qualitative evidence synthesis, based on a literature search which identified 24 papers reporting the findings of 15 population samples (264 individuals with FH and 13 of their family members) across 8 countries (13). Different themes were studied in relation to treatment adherence and as shown in Table 1, six key 
analytical themes were generated, each with specific descriptive themes. These were used to identify seven "enablers" of treatment adherence, including other family members on treatment, commencement of treatment from a young age, receiving a formal diagnosis of $\mathrm{FH}$ and a positive relationship with the HCP. They also identified six barriers to adherence including mismatch between perceived and actual risk, prioritisation of other life events and incorrect and/or inadequate knowledge of treatment advice. These give an important framework for the discussions HCPs should have with both children and young people and their carers about the importance of managing their plasma lipid concentrations and their future CVD risk.

Similar issues were reported in a series of in-depth interviews held with 17 families with a child or young person with a genetic diagnosis of $\mathrm{FH}(14)$. Four main themes were common, including the need for undertaking early prevention, postponing treatment, parental concerns, and the importance of the wider family context. While the majority of parents supported genetic testing for $\mathrm{FH}$ in childhood some were supportive of following early treatment recommendations while others expressed reluctance. Some parents were concerned that inappropriate information had been shared with their children and wished that more time could be allotted to discuss how, when, and what to tell in advance. The authors concluded that additional research is needed to explore the long-term outcomes for children who undertake genetic testing and early treatment for $\mathrm{FH}$, and to test different interventions that may improve the engagement, follow-up, and support of children who are at risk, or diagnosed, with FH.

\section{Genetics and risk factors}

As well as treatment and lifestyle issues, the baseline LDL-C concentration (and thus LDL-C burden and future CVD risk) and to some extent the response to statin therapy and risk of side effects, is determined by the genetic makeup of the child. It is well known that individuals with an LDL-Receptor gene $(L D L R)$ "negative" mutation (eg a partial gene deletion or a single base change that leads to a premature stop codon) have higher untreated LDL-C than those with an LDLR "defective" mutation (eg a missense mutation which retains partial function of the LDL-Receptor). Additionally, those with $\mathrm{FH}$ caused by a mutation in the LDL-C protein Apolipoprotein $B(A P O B)$ have lower mean LDL-C concentrations and show a lower risk of CVD, while mutations in PCSK9 cause a more severe $\mathrm{FH}$ phenotype. Recently it has also been identified that a particular mutation in the gene coding for apolipoprotein $E(A P O E)$, leading to the deletion of one amino acid (p.(Leu167del)), can also mimic the FH phenotype, with these subjects showing a greater LDL-C-lowering response to statin therapy than $L D L R$-FH patients (15).

All guidelines recommend the utility of genetic testing to identify the particular underlying DNA cause for the FH phenotype to support statin treatment and for use in identifying carrier relatives $(4,5,16)$. The utility of genetic testing in $\mathrm{FH}$ has been exemplified by several publications. In a recent paper, two patients from China were studied who were originally clinically diagnosed with Homozygous FH (HoFH), with untreated Total or LDL-C 
over $13 \mathrm{mmol} / \mathrm{L}$. Often individuals with HoFh have a minimal response to statin therapy because they have little or no residual LDL-receptor function that can be upregulated by statin treatment. However, after recording of the family history and genetic testing of the patients and parents, both were found to have a severe form of Heterozygous FH. This was caused by different single nucleotide variants, which caused different changes at the same codon for tryptophan at amino acid 483 in $L D L R$, with the Trp383X mutation, resulting in a premature stop codon and Trp383Gly being a missense mutation. Both patients responded well to conventional statin plus ezetimibe treatment, with LDL-C concentrations being reduced by $67 \%$ and $83 \%$ respectively. This paper demonstrates the clinical utility of having a DNA diagnosis of the underlying disorder and the way in which treatment response can be better predicted.

Even using the most sensitive Next Generation Sequencing approaches, many studies have shown that an $L D L R / A P O B / P C S K 9$ mutation can be found in only $\sim 40 \%$ of patients with a clinical diagnosis of $\mathrm{FH}$. In the remaining 60\%, a "Polygenic" aetiology is most likely to be the cause of the FH phenotype, due to the co-inheritance of common LDL-C raising variants, which are spread throughout the genome, in genes involved in cholesterol metabolism. The clinical utility of a 12-SNP LDL-C "SNP-Score" has been confirmed in samples of no-mutation $\mathrm{FH}$ adults and children from more than eight countries with European-Caucasian populations and most recently from Portugal (18). In a recent analysis of the data (19), it appears likely that in more than $80 \%$ of those with a clinical $\mathrm{FH}$ but with no detectable mutation, the polygenic explanation is the most likely cause of their hypercholesterolaemia. Guidelines now are being modified to take into account that only in families where the index case has a monogenic cause will it be cost effective to carry out cascade testing However, finding a polygenic explanation supports a more conservative (less aggressive) treatment care pathway for adults and children with no mutation.

Satin safety is primarily related to differences in the amount of circulating statin in blood after a standard dose, known as the "systemic exposure". One of the major causes of differences in the systemic exposure is the uptake of statins by the liver, where they have their lipid-lowering action. The SLCO1B1 gene, encoding the solute carrier organic anion transporter family member 1B1 protein, has a common functional variant (c.521T>C, rs4149056) which alters the Valine amino acid at position 174 to Alanine (Val174Ala), which affects the correct localization of the transporter on the basolateral membrane of the hepatocyte. The GnomAD database (https://gnomad.broadinstitute.org/) shows that the frequency of the 174Ala allele is $\sim 16 \%$ in European populations and similar in East Asians, but is much lower in those from the Indian subcontinent (5\%) and in those of African origin (3\%). Thus in European subjects around $27 \%$ will be carriers and $~ 3 \%$ will have two copies of the 174Ala allele. This variant alters the function of the OATP1B1 protein such that those with the 174Ala allele have a much slower clearance of statins to the liver, resulting in higher blood statin levels for longer. Individuals homozygous for the 174Ala allele are known to have an $\sim 17$ fold higher risk of severe myopathy when given simvastatin, with carriers showing a $\sim 4.5$ fold higher risk (20). 
An elegant series of papers has examined the role of the allelic variation in the gene coding for on simvastatin (21) pravastatin (22) and rosuvastatin (23) exposure in children and adolescents (8-20 years old) following administration with a clinically relevant dose. As shown in Figure 3, while the absolute exposure is different with different stains, compared to those with two copies of the 174Val allele, 174Ala carriers have 1.4-2.5 fold higher systemic exposure while Ala/Ala individuals have between 2.2-6.3 fold higher exposure. Simvastatin showed the greatest and rosuvastatin the smallest Val/Val vs Ala/Ala differences. The authors point out that there is still a great deal of inter-individual differences in exposure not explained by this variant, but the extent of this difference is least for rosuvastatin, suggesting that this agent may be more ideal for clinical use in children.

\section{Conclusions}

All published Pediatric FH guidelines recommend the use of genetic tests to confirm the monogenic diagnosis and that statins should be used as the first line of therapy (in tandem with dietary and lifgestyle advice). Consensus among experts proposes consideration of statin initiation by 10 years of age, and particularly for children who have an LDL-C concentration above $3.5 \mathrm{mmol} / \mathrm{l}$. The UK guideline proposes uptitration of statin dose or the addition of ezetimibe in young people over 14 years to lower LDL-C below $2.5 \mathrm{mmol} / \mathrm{I}$. Short term trials of stains show no evidence of clincially significant side effects and no effect on growth or maturation, and one long term follow up confirms both safety and the reduction in CVD in those treated as children as they grow into adulthood. When planned trials of novel LDL-C lowering agents such PCSK9 antibody inhibitors have been completed (24), these agents may also become available to add to the armoury of lipid-lowering therapies to reduce future CVD risk in chidlren with $\mathrm{FH}$.

\section{Key Points}

- For children with FH, statins should be used as the first line of therapy (in tandem with dietary and lifestyle advice).

- Consider statin use by the age of 10 years or earlier if CVD risk factors are present.

- As children approach the age of 14 years, consider to reduce LDL-C below $2.5 \mathrm{mmol} / \mathrm{I}$, by up-titration of statin dose and/or adding other lipid-lowering agents

- Health Care Professionals should have clear and informative discussions with both children and young people and their carers about the importance of managing their plasma lipid concentrations and their future CVD risk.

- Short term trials of stains and long term follow-up show no evidence of clincially significant side effects, no effect on growth or maturation, and the expected reduction in CVD. 


\section{ACKNOWLEDGEMENTS}

Acknowledgements: This work was co-written by SEH and UR.

Financial Support and sponsorship: The authors acknowledge support from the International Atherosclerosis Society (Pfizer number 24052829), the British Heart Foundation (BHF);,HEART UK, Cardiac Network Co-ordinating Group Wales and the Royal College of Physicians. SEH is an Emeritus BHF Professor and acknowledges funding by PG08/008, and by the National Institute for Health Research University College London Hospitals Biomedical Research Centre.

Conflicts of Interest: None 


\section{References}

1. Iyen B, Qureshi N, Kai J, et al. Risk of cardiovascular disease outcomes in primary care subjects with familial hypercholesterolaemia: A cohort study. Atherosclerosis. 2019 Aug;287:8-15

* A retrospective cohort study of 14,097 UK subjects with clinical FH diagnoses matched to 42,506 subjects without FH by age, sex, general practice. During 13.8 years follow-up incidence rates (95\% Cl) of CVD (per 1000 person-years) were 25.6 (24.8-26.3) in FH and 2.9 (2.8-3.1) in non-FH subjects. The risk of CVD was greater in those with $\mathrm{FH}$ characteristics who were in general untreated (HR 13.52, 95\% Cl 12.48-14.65) than those with clinical diagnoses who in general were statin treated (HR 1.66, 95\% Cl 1.421.93).

2. Humphries SE, Cooper JA, Capps N, et al. Coronary heart disease mortality in severe vs. non-severe familial hypercholesterolaemia in the Simon Broome Register Atherosclerosis. 2019 Feb;281:207-212.

** Out of 2929 patients on the register 1982 (67.7\%) patients met the Severe FH definition. The SMR for CHD mortality was significantly $(p=0.007)$ higher for Severe FH (220(184-261) compared to Non Severe FH of 144(98-203). Many of the patients with Severe FH are likely to qualify for treatment with PCSK9 monoclonal antibody inhibitors.

3. Akioyamen LE, Genest J, Shan SD, et al. Estimating the prevalence of heterozygous familial hypercholesterolaemia: a systematic review and meta-analysis. BMJ Open. 2017 Sep 1;7(9):e016461

4. Wiegman A, Gidding SS, Watts GF, et al. Familial hypercholesterolaemia in children and adolescents: gaining decades of life by optimizing detection and treatment. Eur Heart J. 2015 Sep 21;36(36):2425-37

5. Ramaswami U, Humphries SE, Priestley-Barnham L, et al. Current management of children and young people with heterozygous familial hypercholesterolaemia HEART UK statement of care. Atherosclerosis. 2019 Nov;290:1-8.

** Key points are 1) Children with heterozygous familial hypercholesterolaemia (FH) should be managed by paediatric health professionals with expertise in FH. 2) Statin to be considered by the age of 10 years and use age appropriate target LDL-C level in children with FH. 3) Dietary and life style advice should begin in early childhood. 4) From the age of 14 years, consider joint review with adult lipidologist in transition clinic with transfer to adult lipid clinic at 16 to 18 years.

6. Holven KB, Narverud I, van Lennep JR, et al. Sex differences in cholesterol levels from birth to 19 years of age may lead to increased cholesterol burden in females with $\mathrm{FH} \mathrm{J}$ Clin Lipidol. 2018 May - Jun;12(3):748-755.e2.

** By the age of 20 years, the LDL-C burden (Sum of the mean annual LDL-C $x$ age) will be $7.7 \%$ higher in girls $(121.2 \mathrm{mmol} / \mathrm{l}$ in girls and $112.5 \mathrm{mmol} / \mathrm{l}$ in boys). This compares with the LDL-C burden in non FH children of this age of $\sim 45 \mathrm{mmol} / \mathrm{I}$

7. Ramaswami U, Futema M, Bogsrud MP, et al. Comparison of the characteristics at diagnosis and treatment of children with heterozygous familial hypercholesterolaemia (FH) from eight European countries. Atherosclerosis. 2020 Jan;292:178-187.

**Comparison of baseline characteristics and pre and post treatment lipid concentrations in $>3000$ children with FH from 8 countries (Norway, UK, Netherlands, 
Belgium, Czech Republic, Austria, Portugal and Greece). Around 25\% of the treated children and almost $75 \%$ of the untreated children older than 10 years still have LDL-C levels over $3.5 \mathrm{mmol} / \mathrm{l}$, indicating that many children with $\mathrm{FH}$ are not receiving the full potential benefit of early identification and appropriate lipid-lowering treatment according to recommendations.

8. Collins R, Reith C, Emberson J, et al. Interpretation of the evidence for the efficacy and safety of statin therapy. Lancet 2016; 388(10059):2532-61.

9. Vuorio A, Kuoppala J, Kovanen PT, et al. Statins for children with familial hypercholesterolemia. Cochrane Database Syst Rev. 2019 Nov 7;2019(11).

10. Humphries SE, Cooper J, Dale P, et al. The UK Paediatric Familial Hypercholesterolaemia Register: Statin-related safety and 1-year growth data. J Clin Lipidol. 2018 Jan Feb;12(1):25-32.

11. Mamann N, Lemale J, Karsenty A, et al. Intermediate-Term Efficacy and Tolerance of Statins in Children.J Pediatr. 2019 Jul;210:161-165.

12. Luirink IK, Wiegman A, Kusters DM, et al. 20-Year Follow-up of Statins in Children with Familial Hypercholesterolemia. N Engl J Med. 2019 Oct 17;381(16):1547-1556.

** Of the original cohort, 184 of 214 patients with $\mathrm{FH}$ (86\%) and 77 of 95 siblings (81\%) were seen in follow-up. The mean LDL-C concentration in the patients was reduced by $32 \%$ from baseline (from 6.13 to $4.16 \mathrm{mmo} / \mathrm{L}$ ). The cumulative incidence of CVD events and of CVD deaths at age 39 years was lower in children than their affected parents ( $1 \%$ vs. $26 \%$ and $0 \%$ vs. $7 \%$, respectively).

13. Kinnear FJ, Wainwright $E$, Perry $R$, et al. Enablers and barriers to treatment adherence in heterozygous familial hypercholesterolaemia: a qualitative evidence synthesis. BMJ Open. 2019 Jul 31;9(7):e030290.

14. Keenan KF, Finnie RM, Simpson WG, et al. Parents' views of genetic testing and treatment of familial hypercholesterolemia in children: a qualitative study. Community Genet. 2019 Jan;10(1):129-141.

15. Bea AM, Lamiquiz-Moneo I, Marco-Benedí V, et al. Lipid-lowering response in subjects with the p.(Leu167del) mutation in the APOE gene. Atherosclerosis. 2019 Mar;282:143147.

*On high intensity statins, mean reduction in LDL-C significantly higher in 22 (Leu167del) carriers vs 44 LDLR FH $(-52.1 \%$ vs $-40 \%, p=0.040)$. This higher lipidlowering response to statins supports the use of genetics for a more efficient management of $\mathrm{FH}$.

16. Sturm AC, Knowles JW, Gidding SS, et alJ Am Coll Cardiol. 2018 Aug 7;72(6):662-680.

17. Cheng S, Wu Y, Wen W, An M, et al. Independent Severe Cases of Heterozygous Familial Hypercholesterolemia Caused by the W483X and Novel W483G Mutations in the LowDensity Lipoprotein Receptor Gene That Were Clinically Diagnosed as Homozygous CasesGenet Test Mol Biomarkers. 2019 Jun;23(6):401-408.

18. Mariano C, Alves AC, Medeiros AM, et al. The familial hypercholesterolaemia phenotype: Monogenic familial hypercholesterolaemia, polygenic hypercholesterolaemia and other causes. Clin Genet. 2020 Mar;97(3):457-466. 
*731 index patients (311 children and 420 adults) who met the Simon Broome diagnostic criteria. The 6-SNP LDL-C genetic risk score (GRS) for polygenic hypercholesterolaemia was used ( $>25^{\text {th }} \%$ ile $=$ polygenic cause) $\mathrm{An}$ FH-causing mutation was found in $39 \%$ of patients ( $94 \%$ in LDLR, $5 \%$ APOB and $1 \%$ PCSK9), with $29 \%$ with polygenic hypercholesterolaemia

19. Futema $M$, Bourbon $M$, Williams $M$, et al. Clinical utility of the polygenic LDL-C SNP score in familial hypercholesterolemia. Atherosclerosis. 2018 Oct;277:457-463. Wagner JB, Abdel-Rahman S, Van Haandel L, Gaedigk A, Gaedigk R, Raghuveer G, Kauffman R, Leeder JS. Impact of SLCO1B1 Genotype on Pediatric Simvastatin Acid Pharmacokinetics. J Clin Pharmacol. 2018 Jun;58(6):823-833.

20. Link E, Parish S, Armitage J, et al. SLCO1B1 variants and statin-induced myopathy--a genome-wide study. N Engl J Med. 2008 Aug 21;359(8):789-99.

21. Wagner JB, Abdel-Rahman S, Van Haandel et al. Impact of SLCO1B1 Genotype on Pediatric Simvastatin Acid Pharmacokinetics. J Clin Pharmacol. 2018 Jun;58(6):823-833

22. Wagner JB, Abdel-Rahman S, Gaedigk R, et al. Impact of Genetic Variation on Pravastatin Systemic Exposure in Pediatric Hypercholesterolemia.Clin Pharmacol Ther. 2019 Jun;105(6):1501-1512.

23. Wagner JB, Abdel-Rahman S, Gaedigk A, et al. Impact of SLCO1B1 Genetic Variation on Rosuvastatin Systemic Exposure in Pediatric Hypercholesterolemia. Clin Transl Sci. 2020 May;13(3):628-637

** Most recent of 3 papers describing kinetics of statin clearance associated with SCLO1B1 genotype. Also examined CYP2C9 and UGT1A3 genotypes which did not explain interindividual variability seen in the rosuvastatin dose-exposure relationship

24. Gaudet D, Langslet G, Gidding SS, et al. Efficacy, safety, and tolerability of evolocumab in pediatric patients with heterozygous familial hypercholesterolemia: Rationale and design of the HAUSER-RCT study. Clin Lipidol. 2018 Sep - Oct;12(5):1199-1207.

* This paper describes the trial design of use of evolocumab in children with FH but does not present results 
Figures

Figure 1: Standardised Mortality Ratios for CHD deaths of Men and women with Severe FH by age groups (data from (2)

Severe $\mathrm{FH}$ is diagnosed using criteria originally proposed by the International Atherosclerosis Society and are : if the patient has LDL-cholesterol (LDL-C) $>10 \mathrm{mmol} / \mathrm{L}$, or LDL-C >8.0 $\mathrm{mmol} / \mathrm{L}$ plus one high-risk feature, or LDL-C $>5 \mathrm{mmol} / \mathrm{L}$ plus two high-risk features. High-risk features are age $>40$ years without treatment, smoking, male sex, lipoprotein(a) $>75 \mathrm{nmol} / \mathrm{L}$, hypertension, diabetes mellitus, family history of early CHD in first-degree relatives, chronic kidney disease, and $\mathrm{BMI}>30 \mathrm{~kg} / \mathrm{m}^{2}$

Figure 2. UK Consensus guideline for Clinical management of Children and Young People with FH (adapted from 5)

For all children with $\mathrm{FH}$, dietary and lifestyle advice should be discussed at every visit. $\mathrm{CHD}=$ Coronary Heart Disease

* Liver function tests annually and creatine kinase at baseline and thereafter as indicated.

Figure 3. Cartoon showing relative Area under the Curve over 24 hours for statin derivatives in the plasma of children given a standard dose of simvastatin, pravastatin or rosuvastatin, with different SLCO1B1 rs4149056 genotypes. (Data from 21-23)

For full details of the methods used, and dosages of statins employed see the original references. Numbers of children for Simvastatin/Pravastatin/Rosuvastatin with different genotypes were Val/Val (15/15/13) Val/Ala (15/15/13) and Ala/Ala (2/2/2). 